Article

\title{
Cloning and Characterisation of the Gene Encoding 3-Hydroxy-3-Methylglutaryl-CoA Synthase in Tripterygium wilfordii
}

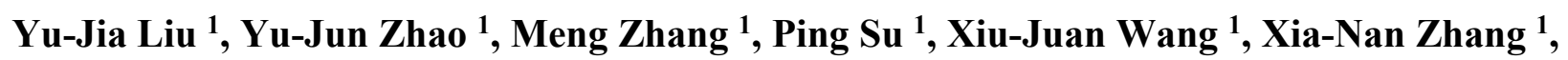 \\ Wei Gao ${ }^{1, *}$ and Lu-Qi Huang ${ }^{2, *}$
}

1 School of Traditional Chinese Medicine, Capital Medical University, Beijing 100069, China; E-Mails: 1yjlife@sina.com (Y.-J.L.); diana1989119@sina.com (Y.-J.Z.); zhangmeng8846@163.com (M.Z.); suping120@163.com (P.S.); wxj0517@sina.com (X.-J.W.); xnzhang@ccmu.edu.cn (X.-N.Z.)

2 National Resource Center for Chinese Materia Medica, China Academy of Chinese Medical Sciences, Beijing 100700, China

* Authors to whom correspondence should be addressed; E-Mails: weigao@ccmu.edu.cn (W.G.); huangluqi01@126.com (L.-Q.H.); Tel.: +86-10-8391-1633 (W.G.); +86-10-6401-4411-2955 (L.-Q.H.); Fax: +86-10-8391-1627 (W.G.); +86-10-6401-3996 (L.-Q.H.).

External Editor: Derek J. McPhee

Received: 27 October 2014; in revised form: 19 November 2014 / Accepted: 20 November 2014 / Published: 27 November 2014

\begin{abstract}
Tripterygium wilfordii is a traditional Chinese medical plant used to treat rheumatoid arthritis and cancer. The main bioactive compounds of the plant are diterpenoids and triterpenoids. 3-Hydroxy-3-methylglutaryl-CoA synthase (HMGS) catalyses the reaction of acetoacetyl-CoA to 3-hydroxy-3-methylglutaryl-CoA, which is the first committed enzyme in the mevalonate (MVA) pathway. The sequence information of HMGS in Tripterygium wilfordii is a basic resource necessary for studying the terpenoids in the plant. In this paper, full-length cDNA encoding HMGS was isolated from Tripterygium wilfordii (abbreviated TwHMGS, GenBank accession number: KM978213). The full length of TwHMGS is $1814 \mathrm{bp}$, and the gene encodes a protein with 465 amino acids. Sequence comparison revealed that TwHMGS exhibits high similarity to HMGSs of other plants. The tissue expression patterns revealed that the expression level of TwHMGS is highest in the stems and lowest in the roots. Induced expression of TwHMGS can be induced by MeJA, and the expression level is highest $4 \mathrm{~h}$ after induction. The functional complement assays
\end{abstract}


in the YML126C knockout yeast demonstrated that TwHMGS participates in yeast terpenoid biosynthesis.

Keywords: HMGS; mevalonate (MVA) pathway; Tripterygium wilfordii; terpenoids

\section{Introduction}

Tripterygium wilfordii is a widely used traditional Chinese medicine that exhibits anti-inflammatory [1] and anti-rheumatoid arthritis [2] activity. Furthermore, bioactive terpenoids from Tripterygium wilfordii were proposed to inhibit growth and induce apoptosis in human cancer cell lines, and have cytotoxic activity [3,4]. The main bioactive chemical compounds of Tripterygium wilfordii belong to different families [5]: triptolide and triptophenolide are diterpenes; celastrol and wilforlide A belong to the triterpenoids. Studies showed that these terpenoids affect cell proliferation in tumours by different mechanisms $[6,7]$ and may provide novel means to treat cancer.

Terpenoids exhibit a broad range of bioactivities and diverse chemical structures. Tanshinones have been extensively used for the treatment of coronary heart disease, cardiovascular disorders, chronic renal failure and human tumours [8], ginkgolides are widely used for the prevention and treatment of thrombi [9], and glycyrrhizic acid exhibits anti-inflammatory, antitumour, antiulcer, antiviral, antiallergic, anti-dotal, and anti-oxidant biological activity [10]. Two terpenoid biosynthetic pathways are commonly recognised in higher plants: the cytosolic mevalonic acid (MVA) pathway [11], and the plastidic 2-methyl-Derythritol-4-phosphate (MEP) pathway [12]. There exists cross-talk between the two pathways [13]. However, the contribution of each pathway in the biosynthesis of different terpenoids is variable [14]. The $\mathrm{C}_{5}$ units from the MEP pathway feed largely into the formation of $\mathrm{C}_{10}, \mathrm{C}_{20}$ and $\mathrm{C}_{40}$ compounds, while the $\mathrm{C}_{5}$ units from MVA pathway feed largely into the formation of $\mathrm{C}_{15}, \mathrm{C}_{25}, \mathrm{C}_{30}$ compounds [15]. HMGS is one of the upstream genes in the MVA pathway that catalyses the formation of the basic $\mathrm{C}_{5}$ building block. HMGS is involved in the catalysis of acetoacetyl-CoA to 3-hydroxy-3-methylglutaryl-CoA, which is the first committed step in the MVA pathway (Scheme 1). The analysis of HMGS in Tripterygium wilfordii is thus important to investigate the biosynthesis of terpenoids active compounds.

Scheme 1. The HMGS-catalyzed chemical reaction.

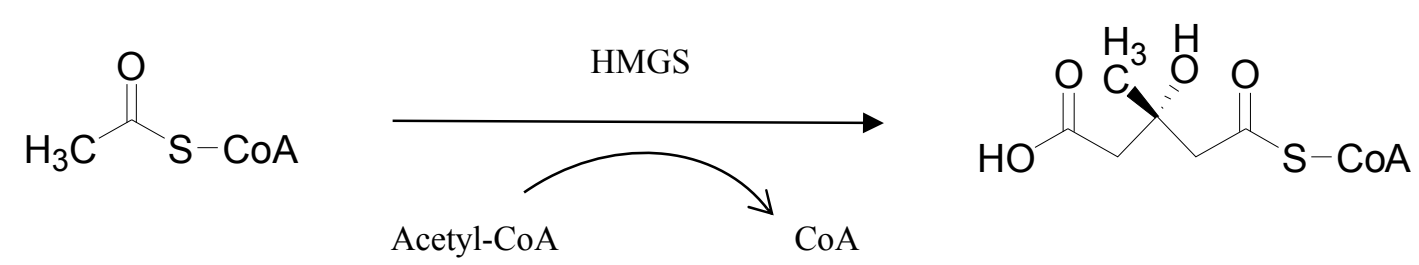

The mechanism of action of HMGS has been widely researched by detecting the structures of its reaction intermediates $[16,17]$. Through the determination of the crystal structure of HMGS bound to different reaction intermediates, the catalytic mechanism of the reaction was determined. This revealed that the carbon-carbon bond formation is facilitated through the activation of the methyl group of an acetylated cysteine [18]. 
However, the enzymes involved in the biosynthesis of terpenoids in Tripterygium wilfordii are not well understood. Because the transcription of HMGS has been proven to be relevant to the accumulation of terpenoids in organisms [19,20], the identification of these enzymes and their genetic sequences are important for further studies of terpenoid biosynthesis in Tripterygium wilfordii. Besides, the sequences of the MVA pathway genes in Tripterygium wilfordii are necessary to produce bioactive compounds in yeast by synthetic biology strategies. To date, no study has described the genes in the MVA pathway of Tripterygium wilfordii. Therefore, we cloned the HMGS gene in Tripterygium wilfordii and characterised the gene using yeast complement assays. In addition, the tissues expression of HMGS in Tripterygium wilfordii aseptic seedlings and the expression in Tripterygium wilfordii suspension cells induced by Methyl jasmonate (MeJA) were investigated.

\section{Results and Discussion}

\subsection{Cloning and Sequence Analysis of TwHMGS}

Full length $T w H M G S$ is 1814 bp and contains a 1398-bp ORF. The gene encodes a 465-amino acid protein. The multiple alignment analysis demonstrated that TwHMGS exhibited high similarity to $H M G S$ genes from other plants, including Panax ginseng (ADI80347.1, 86\%), Hevea brasiliensis (BAF98279.1, 86\%), and Brassica juncea (AAG32924, 80\%) (Figure 1). Based on the functional domain analysis, the active site of HMG-CoA synthase active site exists between amino acids 105 and 120 [18].

The phylogenetic tree was constructed according to the deduced amino acid sequence of $T w H M G S$ and other HMGS genes from different hosts (Figure 2). The tree revealed that TwHMGS exhibited the highest homology with HMGS from Hevea brasiliensis. All of the HMGS genes selected from the plants clustered together, and the HMGS gene from eumycophyta clustered as a different group. 3D modelling by the Swiss-Model (template: Brassica juncea, Seq Identity: 83.93\%) indicated that the TwHMGS catalytic domain was able to form a homodimer with two acetyl-CoA ligands [21] (Figure 3).

\subsection{Functional Identification in Yeast}

The MVA pathway is essential for yeast survival, and the absence of HMGS in yeast is lethal. YSC6274 is a HMGS-disrupted heterozygotic diploid strain that can grow on YPD + G418 medium.

The haploid YSC6274 transfected with empty pYES2 vector was unable to grow on either YPD or YPG medium. The haploid strains transformed with plasmids were unable to grow on SC (-Ura) + 5-FOA medium. The diploid YSC6274 was able to grow on both YPD + G418 and YPG + G418 medium (Figure 4A,C). However, the haploid HMGS-disrupted strain transfected with the pYES2-TwHMGS vector died on YPD+G418 medium (Figure 4B) but was able to survive on YPG + G418 medium (Figure 4D). These data demonstrate that TwHMGS possesses HMGS activity. 
Figure 1. Alignment of $T w H M G S$ with other HMGS genes in plants.

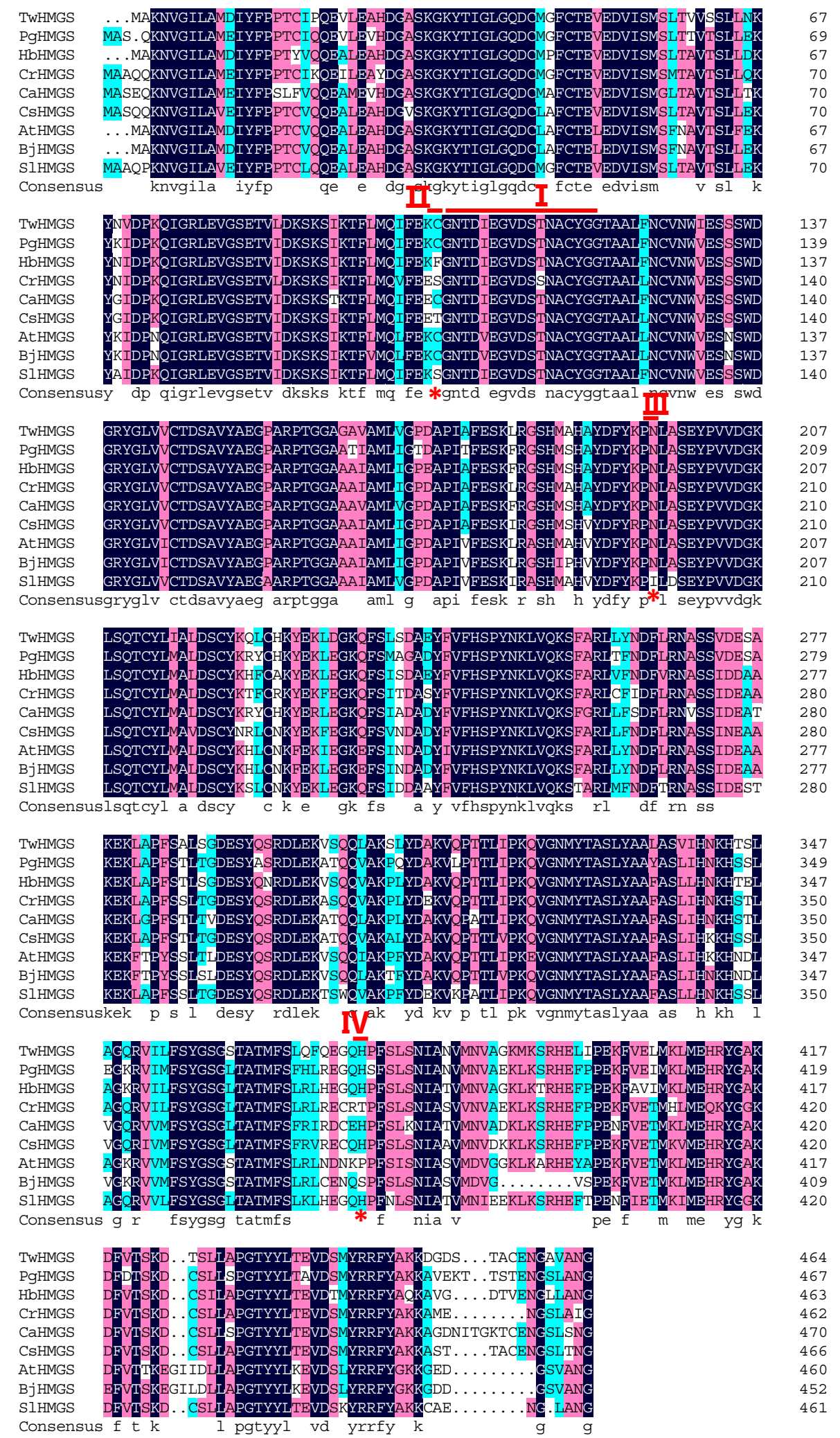

I. The active site of HMGS (105-120); II. Cys amino acid residue; III. Asn amino acid residue; IV. His amino acid residue. * The conserved amino residues. The accession numbers are as follows: TwHMGS (Tripterygium wilfordii, KM978213), PgHMGS (Panax ginseng, ADI80347), HbHMGS (Hevea brasiliensis, BAF98279), CrHMGS (Catharanthus roseus, AEC13715), CaHMGS (Camptotheca acuminate, ACD87446), CsHMGS (Camellia sinensis, AFC34137), AtHMGS (Arabidopsis thaliana, NP_192919), BjHMGS (Brassica juncea, AAG32924), and SlHMGS (Solanum lycopersicum, ABX55778). 
Figure 2. Phylogenetic tree of HMGS from different species using the neighbour-joining method.

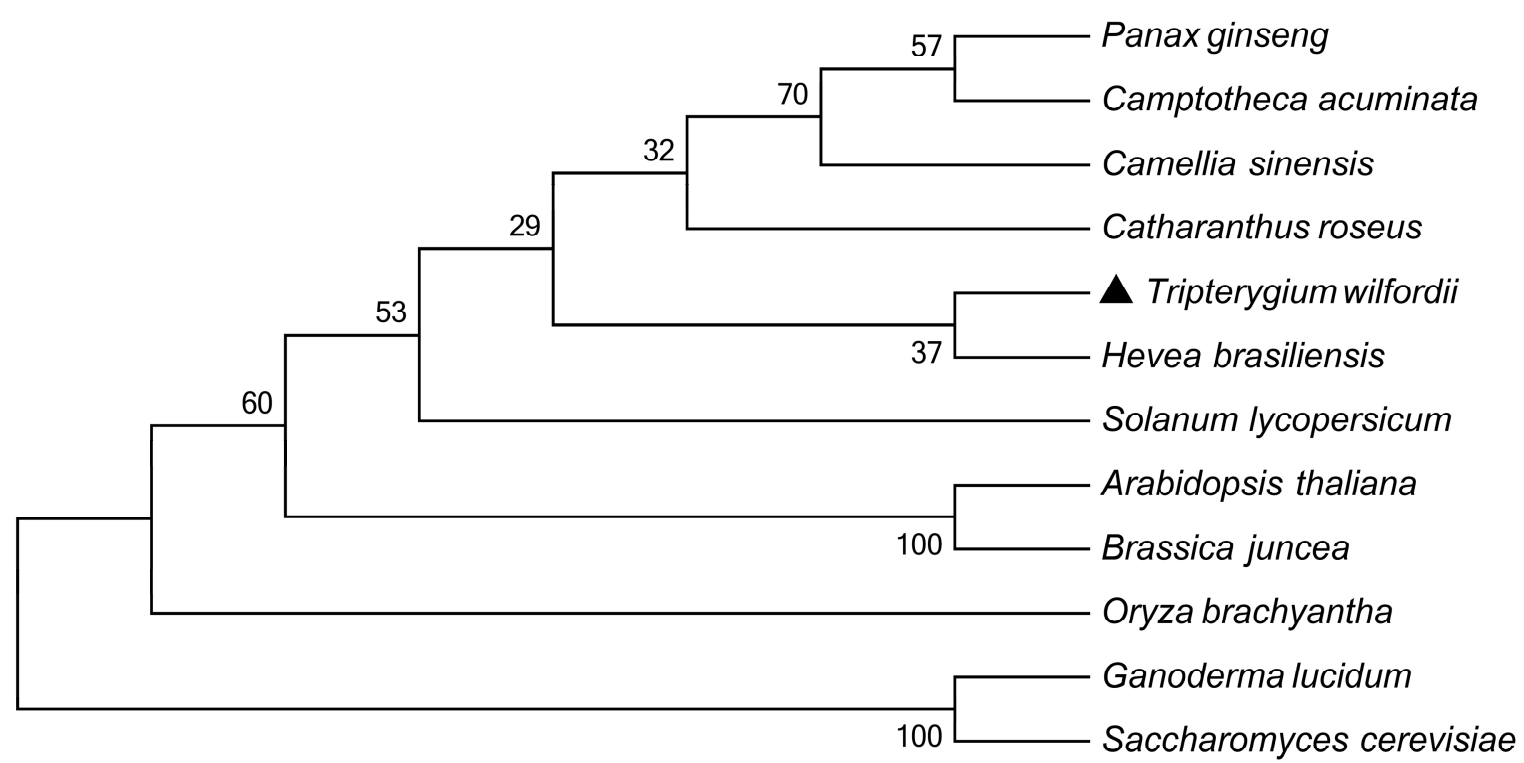

The amino acid sequences were retrieved from the GenBank. $\boldsymbol{\Delta}$ The TwHMGS sequence. The accession numbers are as follows: Tripterygium wilfordii, KM978213; Panax ginseng, ADI80347; Hevea brasiliensis, BAF98279; Catharanthus roseus, AEC13715; Camptotheca acuminate, ACD87446; Camellia sinensis, AFC34137; Arabidopsis thaliana, NP_192919; Brassica juncea, AAG32924; Solanum lycopersicum, ABX55778; Ganoderma lucidum, AFM91095; Saccharomyces cerevisiae, CAA65437; and Oryza brachyantha, ABG73437.

Figure 3. The 3D structure of TwHMGS using homology-based modelling.

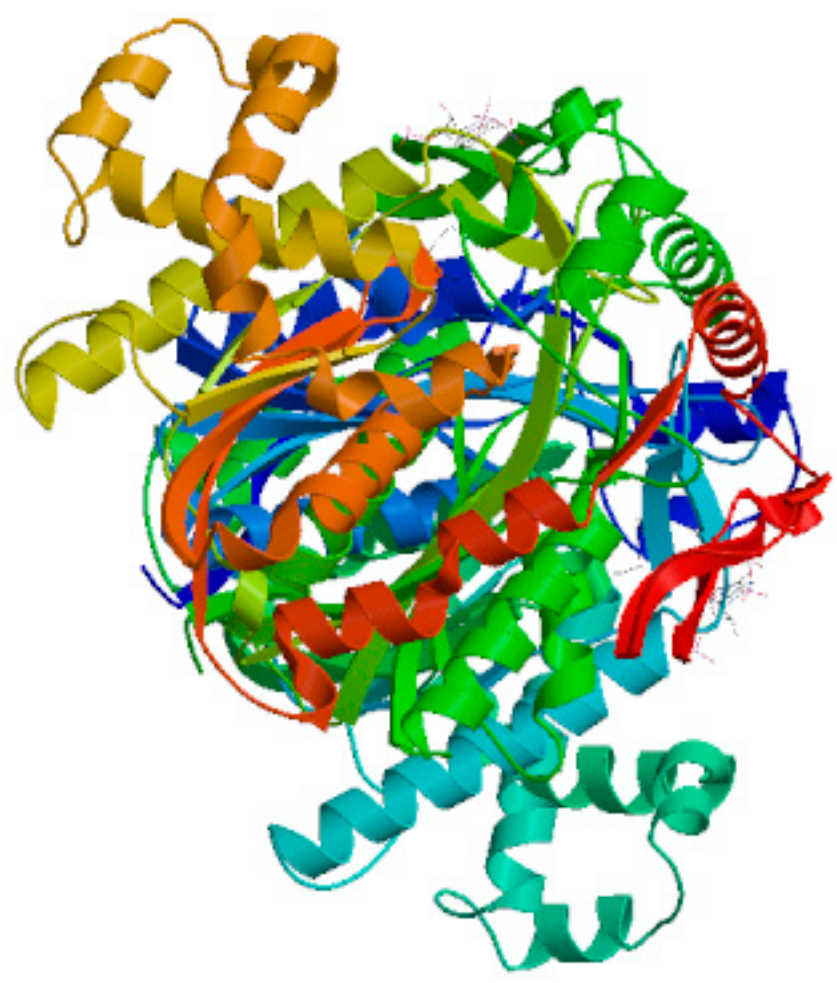


Figure 4. Functional complementation for the growth of the yeast strain YSC6274. (A) Diploid YSC6274 on YPD + G418 medium grew within 2 days; (B) Haploid YSC6274 containing $p Y E S 2-T w H M G S$ on YPD + G418 medium failed to grow; (C) Diploid YSC6274 on YPG + G418 medium grew within 2 days; (D) Haploid YSC6274 containing pYES2-TwHMGS on YPG + G418 medium grew within 3 days.

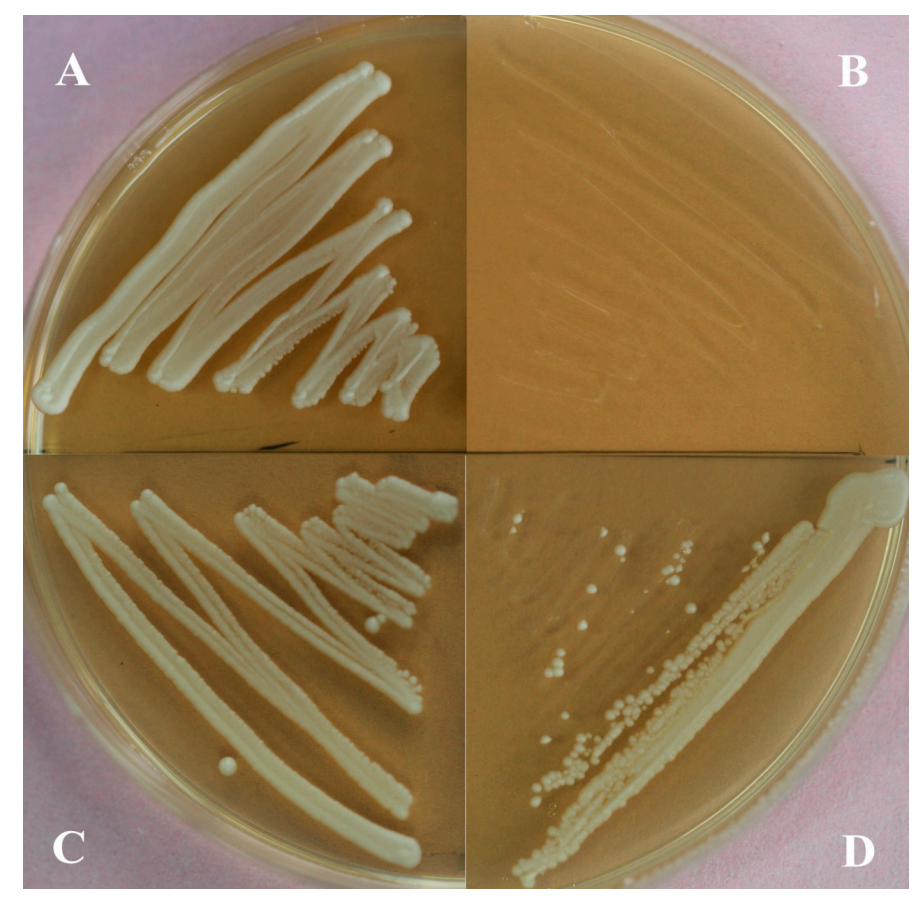

\subsection{Expression of TwHMGS in the Suspension Cells}

Quantitative real-time PCR revealed that TwHMGS expression was induced by $50 \mu \mathrm{M}$ MeJA in suspension cell cultures. The relative expression level of TwHMGS in the induced group reached the highest level at $24 \mathrm{~h}$ after the treatment (21.48-fold that in the control group). However, the expression of TwHMGS was not consistent in the control group; the expression level in the control group was significantly elevated $4 \mathrm{~h}$ after treatment relative to the other time points (Figure 5).

Figure 5. Expression levels of TwHMGS in suspension cells after MeJA treatment.

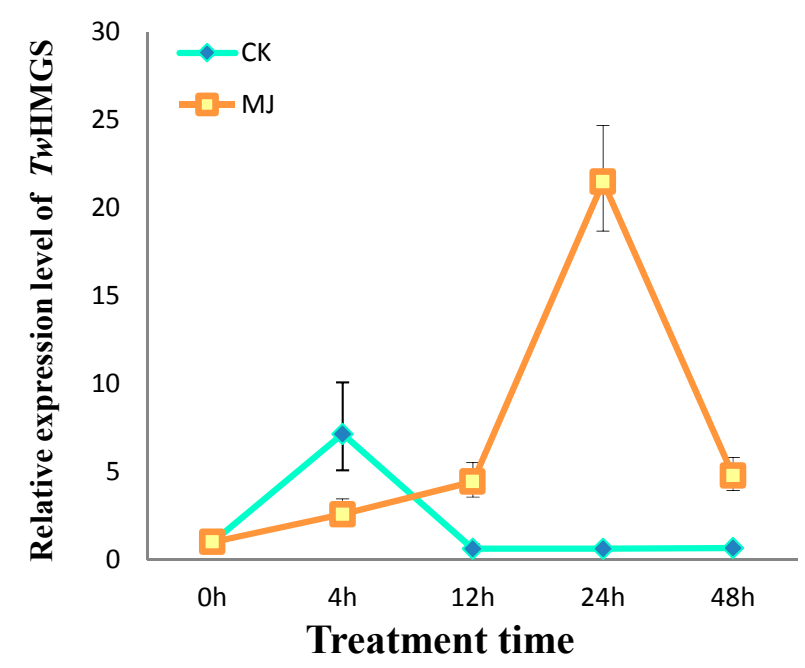

$\mathrm{CK}$, the control group; MJ, the MJ treatment group. 


\subsection{Expression of TwHMGS in Tissues}

TwHMGS was detected in all three kinds of the aseptic T. wilfordii seedling tissues. TwHMGS exhibited the highest expression level in the stem, followed by the leaf. The expression level in the root was the lowest (Figure 6).

Figure 6. Expression of TwHMGS in tissues. (A) The T. wilfordii aseptic seedling and the tissue samples; (B) The relative expression of TwHMGS in different tissues.
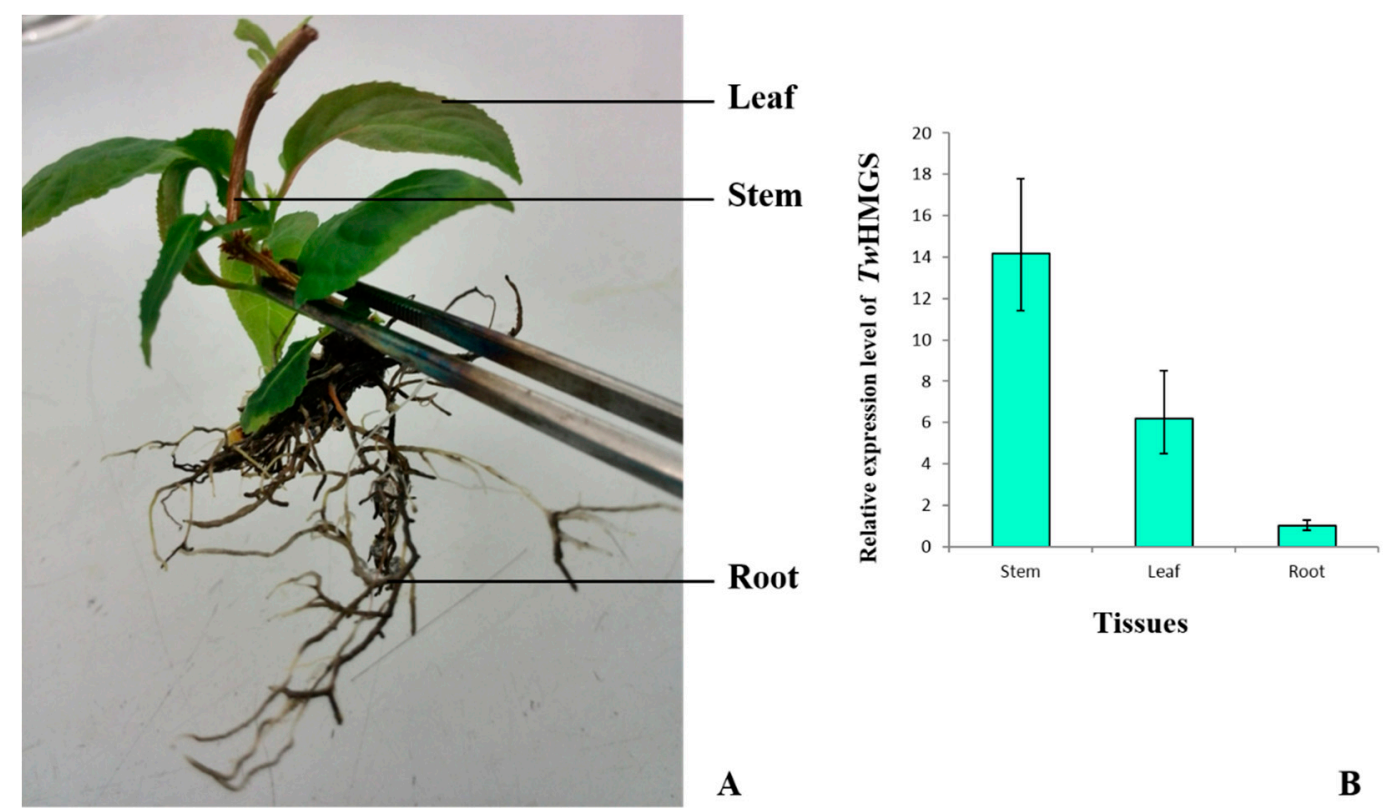

\subsection{Discussion}

Research into the biosynthesis of terpenoids in medicinal plants supports our understanding of the corresponding biosynthetic pathways and provides the possibility of regulating the accumulation of target metabolites. In this study, we examined the terpenoid biosynthesis pathway in Tripterygium wilfordii by first cloning a $H M G S$ gene. The TwHMGS was subsequently transformed into a proper knockout yeast strain to identify the gene function. Furthermore, the induced expression and tissue expression patterns were examined.

Because HMGS is involved in an important step in the MVA pathway, it has been cloned from many plants, including Hevea brasiliensis [22], Taxus media [23], and Arabidopsis thaliana [24]. TwHMGS is highly similar to HMGS sequences from other organisms. Prior catalysis studies have demonstrated that three amino acid residues are absolutely conserved in HMGS: Cys, His, and Asn [25]. These residues are also conserved in TwHMGS. The TwHMGS complement assays revealed that the expression of $T w H M G S$ provides basic material for yeast survival. Gal in the YPG + G418 medium stimulated the expression of TwHMGS, whereas no expression was observed in the YPD + G418 medium due to the absence of Gal in YPD + G418 medium.

The expression pattern of $T w H M G S$ in aseptic seedlings revealed that the gene is expressed in all tissues, but is expressed at higher levels in the stems. Interesting, though the woody stem of Tripterygium wilfordii can be used in traditional medicine, the root is the most commonly used tissue. This result suggest that further studies are needed to investigate the best tissue for medicinal use of the 
plant by chemical quantity analysis. HMGS expression in cell suspensions was induced at $12 \mathrm{~h}, 24 \mathrm{~h}$ and $48 \mathrm{~h}$ after MeJA treatment. The relative expression peaked at $24 \mathrm{~h}$ and fell by $48 \mathrm{~h}$. However, the expression level of the CK group also exhibited a transient increase. This result may reflect expression level changes after treatment with DMSO solvent. With increasing treatment time, the expression level of the CK group was similar with that at $0 \mathrm{~h}$.

In conclusion, the cloning of TwHMGS provides a foundation for further studies of the biosynthesis of terpenoids in Tripterygium wilfordii. The sequence provides a basic gene element for the generation of bioactive terpenoids in Tripterygium wilfordii. Researchers have already produced natural bioactive products in yeast or E. coli using synthentic biology strategies [26,27]. The correlation between $H M G S$ expression and compound occurrence needs to be investigated to produce the bioactive terpenoids on a large scale. Meanwhile, TwHMGS is also a committed enzyme in the biosynthetic pathway engineering of active terpenoids in Tripterygium wilfordii. Further research will be necessary to clarify the process of catalysis in plants to fully understand the biosynthesis of metabolites. And other relevant genes involved in terpenoids synthesis in Tripterygium wilfordii need to be detected.

\section{Experimental Section}

\subsection{Plant Material}

T. wilfordii aseptic seedling cultures were grown in Murashige and Skoog (MS) medium containing $30 \mathrm{~g} / \mathrm{L}$ sucrose and $8 \mathrm{~g} / \mathrm{L}$ agar with $0.2 \mathrm{mg} \cdot \mathrm{L}^{-1} \beta$-indoleacetic acid (IAA), $0.5 \mathrm{mg} \cdot \mathrm{L}^{-1} \mathrm{kinetin}(\mathrm{KT}$ ), and $1.5 \mathrm{mg} \cdot \mathrm{L}^{-1}$ 6-benzylaminopurine (6-BA). The aseptic seedlings were cultured at $25 \pm 1{ }^{\circ} \mathrm{C}$ with $16 \mathrm{~h}$ lighting $/ 8 \mathrm{~h}$ dark. The $T$. wilfordii cell suspensions were cultured in MS medium containing $30 \mathrm{~g} / \mathrm{L}$ sucrose and $8 \mathrm{~g} / \mathrm{L}$ agar with $0.5 \mathrm{mg} \cdot \mathrm{L}^{-1}$ 2,4-dichlorophenoxyacetic acid (2,4-D), $0.1 \mathrm{mg} \cdot \mathrm{L}^{-1} \mathrm{KT}$, and $0.5 \mathrm{mg} \cdot \mathrm{L}^{-1}$ indole-3-butytric acid (IBA). All suspension cell cultures were maintained at $25 \pm 1{ }^{\circ} \mathrm{C}$ with shaking at $120 \mathrm{rpm}$ in the dark.

\subsection{RNA Isolation}

The 10-day-old T. wilfordii suspension cells were treated with MeJA for 0, 4, 12, 24 or $48 \mathrm{~h}$ at a final concentration of $1 \mathrm{mM}$. Subsequently, the suspension cells were harvested for RNA isolation. The root, stem and leaf of the aseptic seedling of $T$. wilfordii were separately harvested for RNA isolation as well. The total RNA was isolated using the cetyltrimethylammonium bromide (CTAB) method [28].

\subsection{Strains}

The HMGS knockout yeast strain YSC6274 (BY4743; MAT a/ $\alpha$; his3D1/his3D1; leu2D0/ leu2D0; lys2D0/LYS2; MET15/met15D0; ura3D0/ura3D0; yml126c: kanMX4/YML126c) was obtained from the GE Healthcare Yeast Knock Out Strain Collection (GE Healthcare Dharmacon, Inc., Lafayette, CO, USA).

\subsection{Cloning of TwHMGS Full-Length cDNA}

Total RNA was reverse transcribed into first-stand cDNA with SMARTer ${ }^{\text {TM }}$ RACE cDNA Amplification Kit (Clontech Laboratories Inc., Mountain View, CA, USA) according to the manufacturer's 
instructions. The full-length primers (HMGS-F: 5'-GATGGACCTTTCTTGTTTCCTCTA-3' and HMGS-R: 5'-GAGAAGCGAAAAATACACTGAAAAC-3') were designed based on the transcriptome sequencing data of Tripterygium wilfordii obtained previously. The product was purified and cloned into the pMD19-T vector (Takara Biotechnology (Dalian) Co., Ltd., Dalian, China). The vector was transformed into E. coli DH5 $\alpha$ cells and cultured in Luria-Bertani medium at $37^{\circ} \mathrm{C}$ in dark. The positive colonies were sequenced and assembled to verify the correct TwHMGS insertion.

\subsection{Bioinformatics Analysis}

The nucleotide sequence was BLAST on NCBI (National Center for Biotechnology Information). The open reading frame (ORF) and amino acid sequence of TwHMGS were deduced using the ORF finder [29]. TwHMGS and other HMGS sequences downloaded from GenBank were aligned, and the phylogenetic tree was constructed by the neighbour-joining method using MEGA5.1. The 3-dimensional structural modelling was predicted by Swiss-Model.

\subsection{Construction of the pYES2-TwHMGS Expression Vector}

The fragment containing TwHMGS ORF and the proper restriction enzyme sites was amplified using HMGS-BamHI (5'-cgcggatccATGGCAAAGAATGTCGGG-3') and HMGS-EcoRI (5'-ccggaattc AGTGACCATTGGCAACTGC-3') as primers. The product and the empty pYES2 vector were digested, gel-purified and ligated. Positive colonies were confirmed by PCR and sequencing. The constructed pYES2-TwHMGS plasmids were extracted and stored at $-20^{\circ} \mathrm{C}$ before transformation into yeast.

\subsection{Complementation Assays}

The glycerol stock of YSC6274 was activated twice on YPD medium (1\% yeast extract, 2\% Bacto-Peptone and $2 \%$ glucose) at $30{ }^{\circ} \mathrm{C}$ in advance. pYES2-TwHMGS was transformed into YSC6274 using the Frozen-EZ Yeast Transformation II Kit (ZymoResearch, Irvine, CA, USA). Meanwhile the empty pYES2 plasmid was also transformed as a control. The transformants were spotted on SC (-Ura) medium ( $6.7 \%$ yeast nitrogen base w/o amino acid, $2 \%$ galactose), and the positive colonies was further verified by PCR. Subsequently, the transformed diploid cells were forced to sporulate and form the haploid cells containing pYES2-TwHMGS. The haploid cells were selected on YPG + G418 medium ( $1 \%$ yeast extract, $2 \%$ Bacto-Peptone, $2 \%$ galactose and $200 \mathrm{ml} / \mathrm{L}$ geneticin). The diploid and the haploid cells were grown on YPD + G418 (1\% yeast extract, 2\% Bacto-Peptone, $2 \%$ glucose and $200 \mathrm{ml} / \mathrm{L}$ geneticin) and YPG + G418 medium separately to compare the grown states. SC (-Ura) + 5-FOA medium (6.7\% yeast nitrogen base w/o amino acid, $2 \%$ galactose and $0.1 \% 5$-fluoroorotic acid) was used to analyse plasmid loss.

\subsection{Quantitative Real-Time PCR}

Total RNA was used to synthesise the first strand cDNA with TIANScript II RT Kit (Tiangen Biotech (Beijing) Co., Ltd., Beijing, China) according to the manufacturer's protocols. The relative mRNA levels were estimated with the Applied Biosystems 7300 Real Time PCR System (Applied Biosystems, Grand Island, NY, USA) using KAPA SYBR ${ }^{\circledR}$ FAST qPCR Kit (KAPA Biosystems, Wilmington, MA, USA). 
The expression of the target gene was normalised according to the expression of the housekeeping gene, $\beta$-actin, and evaluated using the $2^{-\Delta \Delta C t}$ method [30]. There were three samples in each group and each sample was repeated for three times to insure the credibility of the data. The real-time PCR primers were designed by Primer Premier 5.0 as follows: $\beta$-actin F (5'-AGGAA CCACCGATCCAGACA-3') and $\beta$-actin R (5'-GGTGCCCTGAGGTCCTGTT-3'), and HMGS-qF (5'-CTGGAGGTAGGGAGCGAGAC-3') and HMGS-qR (5'-CCATAGCAGGCATTGGTTGA-3').

\section{Conclusions}

In this paper, the HMGS gene in Tripterygium wilfordii was cloned and characterised using yeast complement assays. The TwHMGS presents high similarity with other HMGSs and belong to the HMGS family. The tissues expression of HMGS in Tripterygium wilfordii aseptic seedlings and the expression in Tripterygium wilfordii suspension cells induced by Methyl jasmonate (MeJA) were detected.

\section{Acknowledgments}

This work was supported by the National Natural Science Foundation of China (81422053 and 81373906 to W.G, and 81325023 to L.H.), the Author of National Excellent Doctoral Dissertation of China (201188) and the Project of Construction of Innovative Teams and Teacher Career Development for Universities and Colleges Under Beijing Municipality (CIT\&TCD201304174) to W.G.

\section{Author Contributions}

Lu-Qi Huang and Wei Gao designed research; Yu-Jia Liu, Yu-Jun Zhao, Meng Zhang, Ping Su and Xiu-Juan Wang performed research and analyzed the data; Yu-Jia Liu and Xia-Nan Zhang wrote the paper. All authors read and approved the final manuscript.

\section{Conflicts of Interest}

The authors declare no conflict of interest.

\section{References}

1. Wang, C.; Li, C.J.; Yang, J.Z.; Ma, J.; Chen, X.G.; Hou, Q.; Zhang, D.M. Anti-inflammatory sesquiterpene derivatives from the leaves of Tripterygium. wilfordii. J. Nat. Prod. 2013, 76, 85-90.

2. Marcus, D.M. Comparison of Tripterygium wilfordii Hook F with methotrexate in the treatment of rheumatoid arthritis. Ann. Rheum. Dis. 2014, 73, doi:10.1136/annrheumdis-2013-204807.

3. Zhao, F.; Huang, W.; Ousman, T.; Zhang, B.; Han, Y.; Clotaire, D.Z.; Wang, C.; Chang, H.; Luo, H.; Ren, X.; et al. Triptolide induces growth inhibition and apoptosis of human laryngocarcinoma cells by enhancing p53 activities and suppressing E6-mediated p53 degradation. PLoS One 2013, 8, e80784.

4. Chen, G.; Zhang, X.; Zhao, M.; Wang, Y.; Cheng, X.; Wang, D.; Xu, Y.; Du, Z.; Yu, X. Celastrol targets mitochondrial respiratory chain complex I to induce reactive oxygen species-dependent cytotoxicity in tumor cells. BMC Cancer 2011, 11, doi:10.1186/1471-2407-11-170. 
5. Su, P.; Cheng, Q.; Wang, X.; Cheng, X.; Zhang, M.; Tong, Y.; Li, F.; Gao, W.; Huang, L. Characterization of eight terpenoids from tissue cultures of the Chinese herbal plant, Tripterygium wilfordii, by high-performance liquid chromatography coupled with electrospray ionization tandem mass spectrometry. Biomed. Chromatogr. 2014, 28, 1183-1192.

6. Yan, X; Ke, X.X.; Zhao, H.; Huang, M.; Hu, R.; Cui, H. Triptolide inhibits cell proliferation and tumorigenicity of human neuroblastoma cells. Mol. Med. Rep. 2014, doi:10.3892/mmr.2014.2814.

7. Han, X.; Sun, S.; Zhao, M.; Cheng, X.; Chen, G.; Lin, S.; Guan, Y.; Yu, X. Celastrol stimulates hypoxia-inducible factor-1 activity in tumor cells by initiating the ROS/Akt/p70S6K signaling pathway and enhancing hypoxia-inducible factor-1 $\alpha$ protein synthesis. PLoS One 2014, 9, e112470, doi:10.1371/journal.pone.0112470.

8. Li, Y.G.; Song, L.; Liu, M.; Hu, Z.B.; Wang, Z.T. Advancement in analysis of Salviae miltiorrhizae Radix et Rhizoma (Danshen). J. Chromatogr. A 2009, 1216, 1941-1953.

9. Xie, J.; Ding, C.; Ge, Q.; Zhou, Z.; Zhi, X. Simultaneous determination of ginkgolides A, B, C and bilobalide in plasma by LC-MS/MS and its application to the pharmacokinetic study of Ginkgo biloba extract in rats. J. Chromatogr. B 2008, 864, 87-94.

10. Lu, L.; Zhao, Y.; Yu, H.S.; Huang, H.Z.; Kang, L.P.; Cao, M.; Cui, J.M.; Yu, L.Y.; Song X.B.; Ma, B.P. Preparation of Glycyrrhetinic Acid Monoglucuronide by Selective Hydrolysis of Glycyrrhizic Acid via Biotransformation. Chin. Herbal Med. 2012, 4, 324-328.

11. Laule, O.; Fürholz, A.; Chang, H.S.; Zhu, T.; Wang, X.; Heifetz, P.B.; Gruissem, W.; Lange, M. Crosstalk between cytosolic and plastidial pathways of isoprenoid biosynthesis in Arabidopsis thaliana. Proc. Nat. Acad. Sci. USA 2003, 100, 6866-6871.

12. Chappell, J. Biochemistry and molecular biology of the isoprenoid biosynthetic pathway in plants. Plant Mol. Biol. 1995, 46, 521-547.

13. Denbow, C.J.; Lång, S.; Cramer, C.L. The N-terminal domain of tomato 3-hydroxy-3-methylglutarylCoA reductases. Sequence, microsomal targeting, and glycosylation. J. Biol. Chem. 1996, 271, 9710-9715.

14. Skorupinska-Tudek, K.; Poznanski, J.; Wojcik, J.; Bienkowski, T.; Szostkiewicz, I.; Zelman-Femiak, M.; Bajda, A.; Chojnacki, T.; Olszowska, O.; Grunler, J.; et al. Contribution of the mevalonate and methylerythritol phosphate pathways to the biosynthesis of dolichols in plants. J. Biol. Chem. 2008, 283, 21024-21035.

15. Opitz, S.; Nes, W.D.; Gershenzon, J. Both methylerythritol phosphate and mevalonate pathways contribute to biosynthesis of each of the major isoprenoid classes in young cotton seedlings. Phytochemistry 2014, 98, 110-119.

16. Chun, K.Y.; Vinarov, D.A.; Zajicek, J.; Miziorko, H.M. 3-Hydroxy-3-methylglutaryl-CoA synthase. A role for glutamate 95 in general acid/base catalysis of C-C bond formation. J. Biol. Chem. 2000, 275, 17946-17953.

17. Greenspan, M.D.; Yudkovitz, J.B.; Lo, C.Y.; Chen, J.S.; Alberts, A.W.; Hunt, V.M.; Chang, M.N.; Yang, S.S.; Thompson, K.L.; Chiang, Y.C.; et al. Inhibition of hydroxymethylglutaryl-coenzyme A synthase by L-659,699. Proc. Nat. Acad. Sci. USA 1987, 84, 7488-7492.

18. Theisen, M.J.; Misra, I.; Saadat, D.; Campobasso, N.; Miziorko, H.M.; Harrison, D.H. 3-Hydroxy-3-methylglutaryl-CoA synthase intermediate complex observed in "real-time". Proc. Nat. Acad. Sci. USA 2004, 101, 16442-16447. 
19. Vallett, S.M.; Sanchez, H.B.; Rosenfeld, J.M.; Osborne, T.F. A direct role for sterol regulatory element binding protein in activation of 3-hydroxy-3-methylglutaryl coenzyme A reductase gene. J. Biol. Chem. 1996, 271, 12247-12253.

20. Dooley, K.A.; Millinder, S.; Osborne, T.F. Sterol regulation of 3-hydroxy-3-methylglutaryl-coenzyme A synthase gene through a direct interaction between sterol regulatory element binding protein and the trimeric CCAAT-binding factor/nuclear factor Y. J. Biol. Chem. 1998, 273, 1349-1356.

21. Pojer, F.; Ferrer, J.L.; Richard, S.B.; Nagegowda, D.A.; Chye, M.L.; Bach, T.J.; Noel, J.P. Structural basis for the design of potent and species-specific inhibitors of 3-hydroxy-3-methylglutaryl CoA synthases. Proc. Nat. Acad. Sci. USA 2006, 103, 11491-11496.

22. Sando, T.; Takeno, S.; Watanabe, N.; Okumoto, H.; Kuzuyama, T.; Yamashita, A.; Hattori, M.; Ogasawara, N.; Fukusaki, E.; Kobayashi, A. Cloning and characterization of mevalonate pathway genes in a natural rubber producing plant, Hevea brasiliensis. Biosci. Biotechnol. Biochem. 2008, 72, 2049-2060.

23. Kai, G.; Miao, Z.; Zhang, L.; Zhao, D.; Liao, Z.; Sun, X.; Zhao, L.; Tang, K. Molecular cloning and expression analyses of a new gene encoding 3-hydroxy-3-methylglutaryl-CoA synthase from Taxus media. Biol. Plant. 2006, 50, 359-366.

24. Montamat, F.; Guilloton, M.; Karst, F.; Delrot, S. Isolation and characterization of a cDNA encoding Arabidopsis thaliana 3-hydroxy-3-methylglutaryl-coenzyme A synthase. Gene 1995, 167, 197-201.

25. Price, A.C.; Zhang, Y.M.; Rock, C.O.; White, S.W. Structure of beta-ketoacyl-[acyl carrier protein] reductase from Escherichia coli: negative cooperativity and its structural basis. Biochemistry 2001, 40, 12772-12781.

26. Alper, H.; Miyaoku, K.; Stephanopoulos, G. Construction of lycopene-overproducing E.coli strains by combining systematic and combinatorial gene knockout targets. Nat. Biotechol. 2005, 23, 612-616.

27. Ajikumar, P.K.; Xiao, W.H.; Tyo, K.E.; Wang, Y.; Simeon, F.; Leonard, E.; Mucha, O.; Phon, T.H.; Pfeifer, B.; Stephanopoulos, G. Isoprenoid pathway optimization for taxol precursor overproduction in Escherichia coli. Science 2010, 220, 70-74.

28. Del Sal, G.; Manfioletti, G.; Schneider, C. The CTAB-DNA precipitation method: A common mini-scale preparation of template DNA from phagemids, phages or plasmids suitable for sequencing. Biotechniques 1989, 7, 514-520.

29. ORF Finder (Open Reading Frame Finder). Available online: http://www.ncbi.nlm.nih.gov/ projects/gorf/ (accessed on 25 November 2014).

30. Livak, K.J.; Schmittgen, T.D. Analysis of relative gene expression data using realtime quantitative PCR and the 2- $\Delta \Delta \mathrm{Ct}$ method. Methods 2001, 25, 402-408.

Sample Availability: Samples of the compounds are available from the authors.

(C) 2014 by the authors; licensee MDPI, Basel, Switzerland. This article is an open access article distributed under the terms and conditions of the Creative Commons Attribution license (http://creativecommons.org/licenses/by/4.0/). 